Copyright (C) 2006 IEEE. Reprinted from IEEE Transactions on Geoscience and Remote Sensing, 2006; 44 (9):2421-2429

This material is posted here with permission of the IEEE. Such permission of the IEEE does not in any way imply IEEE endorsement of any of the University of Adelaide's products or services. Internal or personal use of this material is permitted. However, permission to reprint/republish this material for advertising or promotional purposes or for creating new collective works for resale or redistribution must be obtained from the IEEE by writing to pubs-permissions@ieee.org.

By choosing to view this document, you agree to all provisions of the copyright laws protecting it. 


\title{
Modeling and Migration of 2-D Georadar Data: A Stationary Phase Approach
}

\author{
Stewart A. Greenhalgh and Laurent Marescot
}

\begin{abstract}
This paper presents the basic kinematic and dynamic imaging and migration equations for zero-offset two-dimensional georadar profiling. The kinematic equations are derived from simple considerations of spatial impulse responses and a generating function. The dynamic equations follow from a multidimensional stationary phase approximation to the infinite spectral integrals. They show how the radar signal (amplitude and phase) depends on the shape and curvature of the reflector. The imaging equations are evaluated for the special cases of a point scatterer, a continuous reflector, and a terminating reflector. A general formula is developed by which to migrate an arbitrary shaped event of variable amplitude on the georadar section.
\end{abstract}

Index Terms-Georadar, migration, modeling, stationary phase approximation.

\section{INTRODUCTION}

G ROUND penetrating radar (GPR) or georadar surveys are now commonplace in environmental and engineering geophysics for investigating the shallow subsurface [1], [2]. The primary aim is to map the architecture of sedimentary sequences and to delineate geological structures [3], [4]. Most surveys are conducted as two-dimensional (2-D) profiles along straight lines because, unlike seismic surveying, data acquisition is largely undertaken using a single pair of transmitter (Tx) and receiver $(\mathrm{Rx})$ antennas. Multichannel data acquisition is rare; thus, arrays must be synthesized by continually moving the georadar Tx and Rx along the ground surface. The data acquisition is normally coincident $\mathrm{Tx}-\mathrm{Rx}$, at least when profiling. This is referred to as zero-offset. Walkaway or common depth point (CDP) surveys are sometimes run over a range of Tx-Rx offsets to get information on the velocity distribution of the subsurface, but mostly, the velocity is assumed to be constant.

Three-dimensional (3-D) surveys entail an areal distribution of Tx-Rx points on the ground surface. The data are still collected as a series of closely spaced, parallel 2-D lines, to form the grid. This generally involves recording on a single channel at a time and building up each line in turn [5]-[7]. Such field practice is quite distinct from 3-D seismic, in which hundreds or even thousands of recording channels simultaneously record the signals from each source point on multiple Rx lines. Three-

Manuscript received November 16, 2005; revised February 24, 2006. This work was supported by grants from the Australian Research Council and the Swiss Federal Institute of Technology Zürich.

S. A. Greenhalgh is with the Department of Physics, University of Adelaide, 5005 Adelaide, Australia (e-mail: stewart.greenhalgh@adelaide.edu.au).

L. Marescot is with the Institute of Geophysics, Swiss Federal Institute of Technology, 8093 Zurich, Switzerland (e-mail: laurent@aug.ig.erdw.ethz.ch).

Digital Object Identifier 10.1109/TGRS.2006.875363 dimensional georadar data are processed as a full volume to enable the correct positioning of reflections from out of the plane of each 2-D line. Because of the field-time-intensive nature of 3-D georadar surveys, reconnaissance 2-D profiling is still an essential part of any GPR investigations.

In addition to surface profiling, borehole radar experiments are also routinely carried out in support of civil and mining engineering projects [8]. Crosshole surveys, in which the radar Tx is placed in one borehole and the Rx in another, seek to scan the interwell medium in a tomographic sense to image changes in the electromagnetic (EM) wavespeed or attenuation [9]. Borehole profiling utilizes a single (bistatic) radar probe in which $\mathrm{Tx}$ and $\mathrm{Rx}$ modules are separated to interrogate the space surrounding the well. It relies on backscatter or reflection to detect and echolocate geological targets like ore bodies and faults/fractures. Often, several wells are profiled in turn.

In either surface radar profiling or borehole GPR reflection profiling, the data comprise a series of radargrams assembled into what is called a georadar section. The horizontal axis $x$ represents position along the ground surface or distance along the borehole where the Tx-Rx was placed, and the vertical axis is the two-way time. The latter can be converted to a depth or range $z$ by multiplying by half the average wavespeed $V$ of the medium. Without directional antennas, there is a rotational ambiguity with respect to the direction from which the echo has come. Except in 3-D areal surveying or multiple borehole profiling, it is generally assumed that the reflection lies in the sagittal plane formed by the radar profile and its normal. In the case of a surface survey, this will be the vertical $X-Z$ plane.

The radar section bears a deceptive similarity to a geological section. If the geology (object space, $X-Z$ ) is complex, the radar section (image space, $x-z$ ) will be even more complex. There are artifacts of a structural nature (geometry, diffraction hyperbolas, bow ties), well known to seismic interpreters. This is illustrated in Fig. 1 for the case of a tight syncline having its center of curvature beneath the Earth's surface. There are reflections from both sides as well as the base of the syncline, resulting in ray path crossings and a complicated triple image or bow tie pattern. The main branch of the triplication is actually anticlinal. There are also artifacts of a dynamic nature (amplitude changes, phase changes, caustics). To properly interpret the data, it is essential to elucidate the nature of the signal. The technique of migration, adapted from seismic processing [10], [11], is used to unravel some of the artifacts (e.g., collapse diffraction hyperbolas) and to position reflectors properly in their true spatial position. Most radar migration algorithms operate directly on the data in a summation sense (some sort of focused lag-sum operator) and can be carried out using 


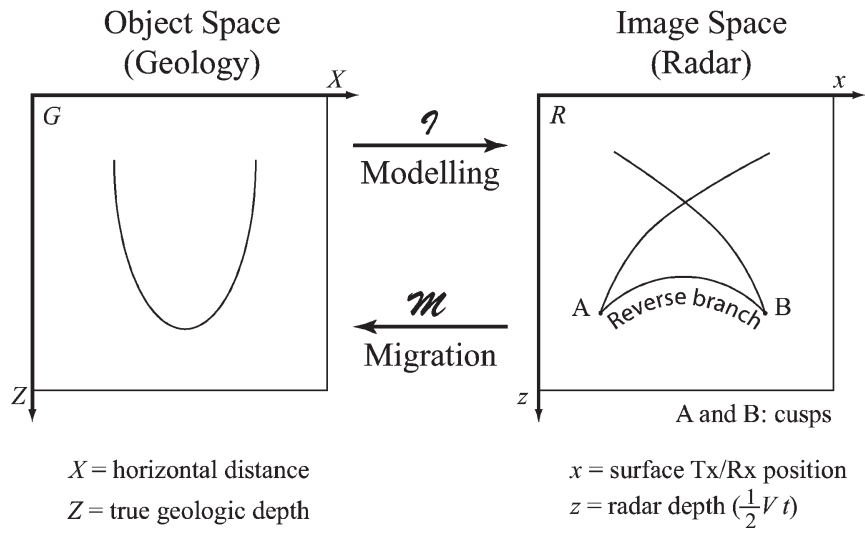

Fig. 1. Object space (geology $G$ ) and image space (radar section $R$ ) and the transformations going from one to the other. $I$ stands for modeling or imaging, or going from $G$ to $R$. The inverse process of moving from $R$ to $G$ is called migration $M$.

finite difference or boundary integral techniques such as the Huygens-Kirchhoff diffraction stack [12]-[14]. Although some vector GPR processing techniques have been developed [15], most georadar migration (and modeling) schemes have their basis in the scalar wave equation, i.e.,

$$
\nabla^{2} u=\frac{1}{V^{2}(X, Y, Z)} \frac{\partial^{2} u}{\partial t^{2}}
$$

where $u$ is the wavefield (e.g., electric field) and $V=1 / \sqrt{\varepsilon \mu}$ is the EM wavespeed, which depends on the permittivity $\varepsilon$ and the permeability $\mu$ of the medium.

The migration technique can itself amplify noise and cause distortions. When the signal-to-noise ratio is low, it is often preferable not to migrate in an automatic full-wavefield sense, but rather to perform only a section-style migration to get the correct geometry of reflectors [16].

In this paper, we develop a simple spectral theory of 2-D radar modeling and migration, which is applicable to constant velocity media. If the velocity actually varies, then one must use the average velocity above the reflector in question. We apply the method of stationary phase to evaluate the infinite spectral integrals, which is equivalent to a Fresnel approximation. From the simple spectral theory, the basic imaging equations for an arbitrary shaped reflector emerge; they give the transformations from image space to object space, and vice versa. The dynamic equations include amplitude and phase information and show how it depends on reflector curvature. In Section II, we look at spatial impulse responses and imaging kinematics and derive the basic transformation equations from elementary considerations of generating functions. The independent kinematic treatment yields the same equations for image space to object space conversion but is lacking in terms of the actual pulse shape covered in the more elaborate dynamic treatment. The analysis given provides considerable insight into the radar imaging problem and a possible scheme for noisy data migration. It would be a fairly simple matter to extend the analysis to 3-D, but handling inhomogeneous media presents considerable difficulty.

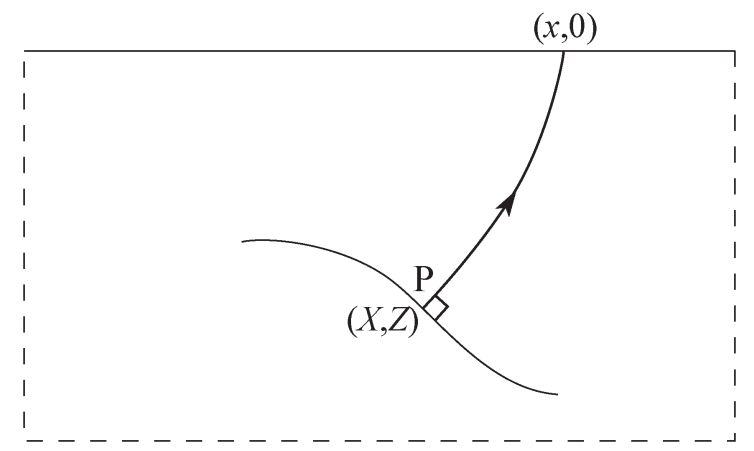

Fig. 2. Coordinates in the two domains. The radar Tx at point $(x, 0)$ sends down a pulse along the normally incident ray path of length $z$ as shown and produces a reflection from the point $(X, Z)$ on the reflector in object space. The echo returns along the same path where it is recorded on the Earth's surface by the radar Rx, also at $(x, 0)$.

\section{Spatial IMPUlse Responses}

Consider the situation depicted in Fig. 2. The radar Tx-Rx is located on the Earth's surface at position coordinate $(x, 0)$. The reflection from the point $(X, Z)$ on the interface follows the curved ray path as shown. The path is curved because of Snell refraction associated with an increase of velocity with depth. The ray strikes the interface at normal incidence at the point $P$ and is of a length $z$, where $z=V t / 2$. Here, $V$ is the average velocity of the radar wave above the interface, and $t$ is the two-way time of the reflection (strictly speaking, $z$ should be expressed as a line integral). The reflected event is plotted at position coordinate $(x, z)$ in the image space. The "image space," which is the radar section, will be denoted $R$. It is quite distinct from the "object space" (viz., the geology), which will be denoted by $G$. The reflection originates from the point $(X, Z)$ in object space. We are interested in the transformation between image space $R$ and object space $G$ (see Fig. 1).

We can think of a generating function $W$, which accomplishes this transformation. It is given by

$$
W(X, Z, x, z)=0 .
$$

In homogeneous media, the velocity is constant and the ray path is a straight line; thus, from simple geometry, we obtain

$$
W=(X-x)^{2}+Z^{2}-z^{2}=0 .
$$

Let us first consider the spatial impulse response for radar modeling. This is the process of going from the object space $G$ to the image space $R$. We are interested in what happens to the point scatterer $\left(X_{0}, Z_{0}\right)$ as shown in Fig. 3(a). Substituting into (3), we obtain for the Cartesian equation of the image, i.e.,

$$
z^{2}-\left(x-X_{0}\right)^{2}=Z_{0}^{2} .
$$

This is the equation of a hyperbola having apex $\left(X_{0}, Z_{0}\right)$, as shown in Fig. 3(a). Thus, the point in object space has become a curve in image space. This is the well-known diffraction hyperbola. In other words, a single point (impulse function) in the object space becomes a hyperbola in the image space. This is the impulse response for radar modeling. 


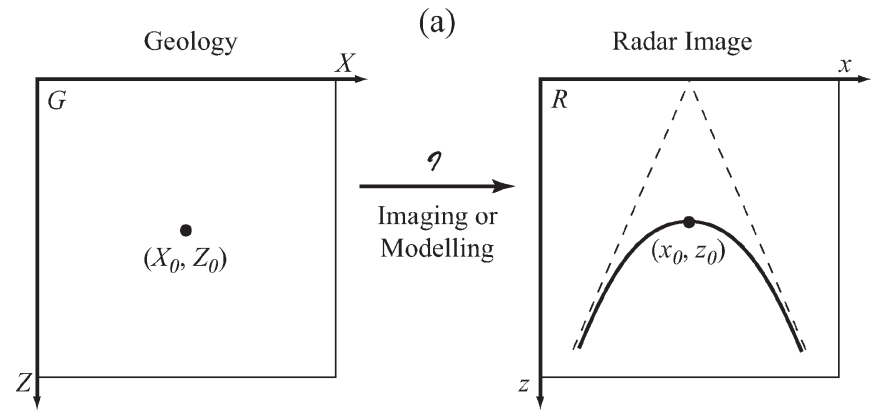

(b)
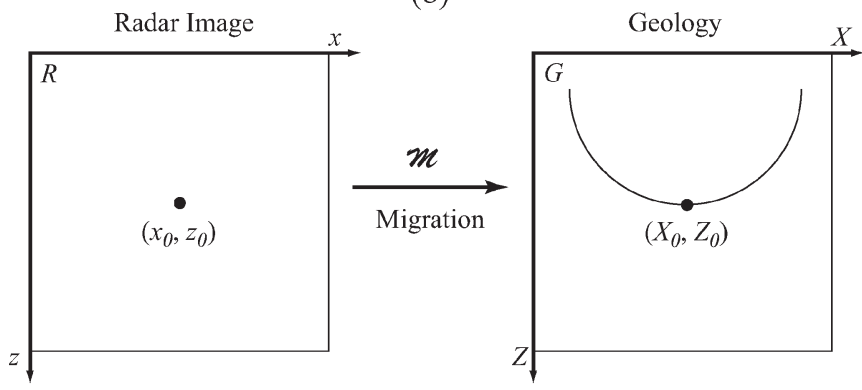

Fig. 3. Spatial impulse responses for (a) modeling (the diffraction hyperbola) and (b) migration (the semicircular wavefront).

We now consider the reverse situation of inverse modeling or "migration," i.e., moving from image space $R$ to object space $G$ [see Fig. 3(b)]. The single point coordinate $\left(x_{0}, z_{0}\right)$ in image space can be substituted into (3) to obtain the corresponding equation in object space, i.e.,

$$
\left(X-x_{0}\right)^{2}+Z^{2}=z_{0}^{2} .
$$

This is the equation of a circle of center $\left(x_{0}, 0\right)$ and radius $z_{0}$; it represents the "wavefront" migration impulse response. Noise spikes in the image space get dispersed into semicircular "smiles" in the migrated section (i.e., object space).

\section{IMAGING KINEMATICS}

\section{A. Modeling a Continuous Reflector}

Having established the basic impulse responses for modeling and migration, we now wish to examine the kinematic modeling equations for an arbitrary shaped continuous reflector. We will later examine the dynamic modeling response. In Cartesian form, the reflector is given by

$$
Z=F(X) .
$$

It has parametric equations $X=X(p), Z=Z(p)$. The generating function can be written as

$$
W(X, F(X), x, z)=(X-x)^{2}+F(X)^{2}-z^{2}=0 .
$$

Referring to Fig. 4, we have a one-parameter family of diffraction hyperbolas. Each point making up the continuous reflector transforms to a hyperbola, the envelope of all such hyperbolas can be found by setting

$$
W=0 \quad \partial W / \partial X=0 .
$$

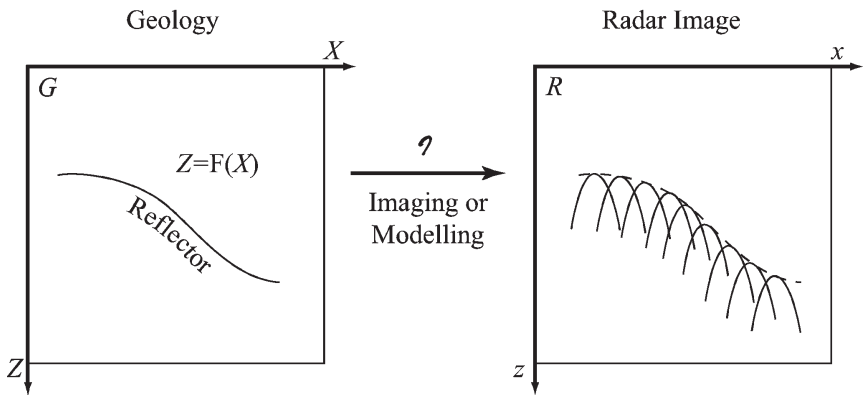

Fig. 4. Modeling a continuous reflector. The image is the superposition (or envelope) of all diffraction hyperbolas from each point in object space. In other words, the reflector is modeled as a continuum of point diffractors.

This yields the parametric equations of the radar image, i.e.,

$$
\begin{aligned}
& x=X+F(X) \cdot F^{\prime}(X) \\
& z=F(X) \sqrt{1+F^{\prime}(X)^{2}}
\end{aligned}
$$

where $F^{\prime}(X)=d F / d X$ is the local geological dip.

The local dip of the radar section is given by

$$
\frac{d z}{d x}=\frac{d z / d X}{d x / d X}=\frac{F^{\prime}(X)}{\sqrt{1+F^{\prime}(X)^{2}}} .
$$

A "caustic point," such as points $A$ and $B$ in Fig. 1, occurs at $\left(x_{0}, z_{0}\right)$ if $d x / d X=d z / d X=0$ at $\left(x_{0}, z_{0}\right)$. Taking the derivative of (8), this yields the condition

$$
\rho=\left(1+F^{\prime}(X)^{2}\right)^{3 / 2} / F^{\prime \prime}(X)=-z
$$

where $F^{\prime \prime}$ is the second derivative and $\rho$ is the radius of curvature of the interface at this point. In other words, a caustic occurs whenever the center of curvature lies beneath the Earth's surface.

\section{B. Migrating a Continuous Horizon}

Consider an arbitrary shaped radar reflector event in image space, which is given by the Cartesian equation

$$
z=h(x) .
$$

The generating function is

$$
W(X, Z ; x, h(x))=(X-x)^{2}+Z^{2}-h(x)^{2}=0 .
$$

This defines a one-parameter family of semicircular wavefronts (see Fig. 5) whose envelope is given by

$$
W=0 \quad \partial W / \partial x=0 .
$$

Substituting into (12), we obtain the following parametric equations for the object space or migrated image:

$$
\begin{aligned}
X & =x-h(x) \cdot h^{\prime}(x) \\
Z & =h(x) \sqrt{1-h^{\prime}(x)^{2}}
\end{aligned}
$$




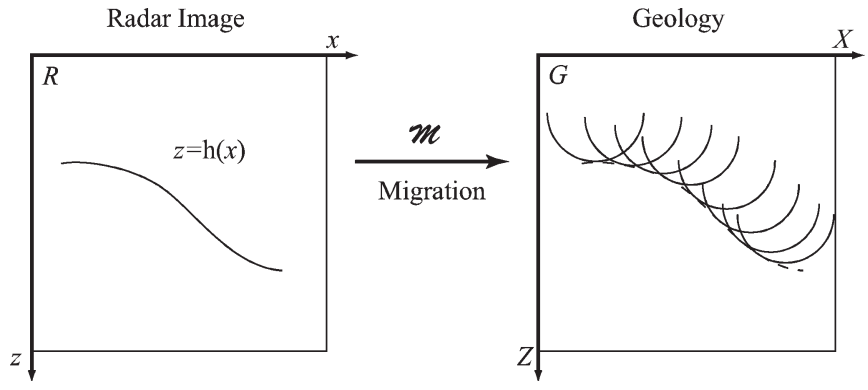

Fig. 5. Migrating a continuous horizon. The final image is the envelope of all semicircular wavefronts. Each point in image space forming the continuous line is dispersed along its corresponding circular wavefront in object space.

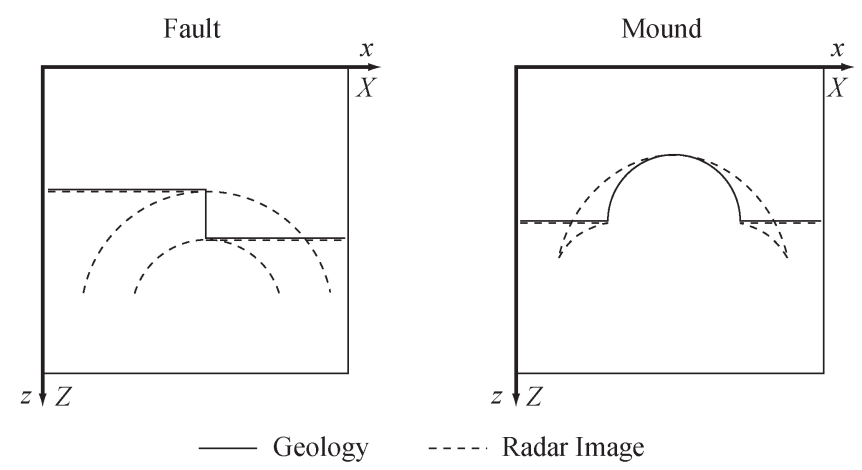

Fig. 6. Object space (geology) representation of a fault structure and a mound structure as well as their image space representation. Note how diffractions in the image space heal discontinuities in the object space.

where $h^{\prime}(x)=d h(x) / d x=\tan \delta$ is the local dip of the radar image. The true geologic dip $\Delta$ is given by

$$
\frac{d Z}{d X}=\tan \Delta=\frac{d Z / d x}{d X / d x}=\frac{h^{\prime}(x)}{\sqrt{1-h^{\prime}(x)^{2}}}
$$

It follows from simple trigonometry that

$$
\tan \delta=\sin \Delta
$$

which is the well-known Migrator's equation. Note that the radar dip $\delta$ is always less steep than the true dip $\Delta$. Whereas $\Delta$ can cover the full range from $-\pi / 2$ to $+\pi / 2$, the radar dip is limited $(|\delta| \leq \pi / 4)$.

Equations (8) and (14) constitute the kinematic imaging equations, which give the connection between the shape of an event in the radar and geologic domains. For example, horizontal interfaces remain horizontal in both image space and object space. Dipping beds are partially flattened on the radar section (16). Anticlines are always broader on the radar section than in reality (although the apex occurs at the same position in both domains), whereas synclines always appear narrower (see Section V-B). Discontinuities in object space are "healed" by diffractions and appear as continuous structures on the radar image. The diffraction effect is illustrated schematically for both a vertical step (fault) and a mound structure in Fig. 6.

\section{SPECTRAL Representation OF DYNAMICAL IMAGING}

Our starting point for coincident $\mathrm{Tx}-\mathrm{Rx}$ georadar modeling is the exploding reflector model in which all points on each interface are assumed to "fire" in synchronism at zero time. The wavefield then evolves upward toward the surface. We convert from two-way time $t$ to pseudo depth $z$ by multiplying by half the velocity $V / 2$. This exploding reflector model has proven particularly useful in CDP seismic imaging [11].

Let $u(X, Z, 0)$ be the wavefield (or representation of the geological reflectors) at zero time. This wavefield is the convolution of the source waveform $g(z)$ with the object space $v(x, Z, z)$. The wavefield $u(x, Z, z)$ at subsequent times and positions can be obtained as the solution of an initial value problem (IVP), i.e.,

$$
\frac{\partial^{2} u}{\partial z^{2}}=\frac{\partial^{2} u}{\partial x^{2}}+\frac{\partial^{2} u}{\partial Z^{2}}
$$

The radar image is obtained by setting $Z=0$ to yield $u(x, 0, z)$, which is the wavefield at the ground surface.

The solution of (17) is best accomplished in the spectral domain. We first take the 2-D Fourier transform of the geology or object space $v(X, Z, 0)$, i.e.,

$$
V(k, \omega, 0)=\int_{-\infty}^{\infty} \int_{-\infty}^{\infty} v(X, Z, 0) \cdot \exp (-i(k X-\omega Z)) d X d Z
$$

with

$$
U(k, \omega, 0)=V(k, \omega, 0) G(\omega)
$$

where $G(\omega)$ is the source spectrum.

The 2-D spectrum at depth $z$ is easily obtained by taking the 2-D Fourier transform of (17) and solving the resulting simple ordinary differential equation, i.e.,

$$
U(k, \omega, z)=U(k, \omega, 0) \cdot \exp \left(-i \sqrt{\omega^{2}+k^{2}} z\right) .
$$

The full wavefield is then obtained by taking the 2-D inverse Fourier transform of $U(k, \omega, z)$, i.e.,

$$
\begin{aligned}
u(x, Z, z)= & \frac{1}{4 \pi^{2}} \int_{-\infty}^{\infty} \int_{-\infty}^{\infty} U(k, \omega, 0) \\
& \cdot \exp \left(i\left(k x-\omega Z-\sqrt{\omega^{2}+k^{2}} z\right)\right) d \omega d k
\end{aligned}
$$

Finally, the image is given by setting $Z=0$, i.e.,

$$
\begin{aligned}
& u(x, 0, z)=\frac{1}{4 \pi^{2}} \int_{-\infty}^{\infty} \int_{-\infty}^{\infty} U(k, \omega, 0) \\
& \cdot \exp \left(i\left(k x-\sqrt{\omega^{2}+k^{2}} z\right)\right) d \omega d k
\end{aligned}
$$


This equation allows us to model the radar response for arbitrary geology, expressed in terms of the 2-D spatial Fourier transform of the object.

\section{A. Stationary Phase Image of a Continuous Reflector}

We will now apply (21) to compute the image of a continuous reflector $Z=F(X)$ for which the reflectivity $S(X)$ varies along its length. We write the geology in the form

$$
v(X, Z, 0)=S(X) \delta(Z-F(X))
$$

where $\delta$ is the Dirac delta function. The 2-D transform reduces to

$$
V(k, \omega, 0)=\int_{-\infty}^{\infty} S(X) \cdot \exp (-i(k X-\omega F(X))) d X .
$$

Changing variables from $k$ to $p$ through the relationship

$$
k=p \omega
$$

we obtain by substituting into (21)

$$
\begin{aligned}
u(x, 0, z)=g(z) & * \frac{1}{4 \pi^{2}} \int_{-\infty}^{\infty}|\omega| d \omega \\
& \cdot \int_{-\infty}^{\infty} \int_{-\infty}^{\infty} S(X) \exp (-i \omega \phi(p, X)) d p d X
\end{aligned}
$$

where $*$ denotes convolution with the source signal $g(z)$, and the phase factor $\phi$ is given by

$$
\phi(p, X)=-p(x-X)+\sqrt{1+p^{2}} z-F(X) .
$$

To proceed, we have to evaluate the infinite integrals. We will use the method of multidimensional stationary phase to do so [17]-[19]. This is an asymptotic solution, which recognizes that the integrand of (25) is highly oscillatory; thus, most of the contribution to the integral (constructive summation) comes from positions of stationary phase. These are points at which the derivative of $\phi$ is zero.

The solution is [20]

$$
u(x, 0, z)=g(z) *|\xi|^{-1 / 2} S(X) \hat{\delta}(\phi(p, X))
$$

where

$$
\begin{aligned}
\hat{\delta}(z) & =\delta(z), \quad \text { if } \xi>0 \\
& =\delta_{H}(z), \quad \text { if } \xi<0
\end{aligned}
$$

and the subscript $H$ denotes Hilbert transformation. The amplitude factor $\xi$ is related to the radius of curvature $\rho$ through the relations

$$
\begin{aligned}
& \xi=\left[1+\frac{z}{\rho}\right] \\
& \rho=\frac{\left(1+F^{\prime}(X)^{2}\right)^{3 / 2}}{F^{\prime \prime}(X)} .
\end{aligned}
$$

Now $(p, X)$ is a stationary point of $\phi(p, X)$ if

$$
\begin{aligned}
& \frac{\partial \phi}{\partial p}=-(x-X)+\frac{p z}{\sqrt{1+p^{2}}}=0 \\
& \frac{\partial \phi}{\partial X}=p-F^{\prime}(X)=0 .
\end{aligned}
$$

At $\phi=0$, we have

$$
\begin{aligned}
& x=X+F(X) F^{\prime}(X) \\
& z=F(X) \sqrt{1+F^{\prime}(X)^{2}}
\end{aligned}
$$

or in Cartesian form, $z=h(x)$. This is the same result as we obtained previously from simple kinematic considerations (8). Instead of the parametric form, we can write (31) in Cartesian form as $z=h(x)$. Thus, $\phi=0$ along $z=h(x)$ and

$$
\delta(\phi)=\phi_{z}^{-1} \delta(z-h(x))
$$

with

$$
\phi_{z}=\frac{d \phi}{d z}=\sqrt{1+F^{\prime}(X)^{2}}
$$

Thus, the stationary phase image of a continuous reflector is given by

$$
u(x, 0, z)=\frac{S(X)}{\sqrt{1+F^{\prime}(X)^{2}}}|\xi|^{-1 / 2} \hat{g}(z-h(x)) .
$$

Consider the special case of a planar dipping reflector, viz., $Z=F(X)=A X+B$, having constant reflectivity $S(X)=1$. It follows that $F^{\prime}(X)=A$ (a constant), $F^{\prime \prime}(X)=0$, and $\rho=\infty$, giving $\xi=1$; thus, the radar image (31), (33) is also a plane dipping event $z=a x+b$ where

$$
a=A /\left(1+A^{2}\right)^{1 / 2} \quad b=B /\left(1+A^{2}\right)^{1 / 2}
$$

of uniform amplitude $1 /\left(1+A^{2}\right)^{1 / 2}$ and uniform pulse shape $g(z)$.

A semicircular basinal reflector, $Z^{2}+(X-R)^{2}=R^{2}$, is also of interest. It leads to the parametric equation (31) for the image of $x=R, z=R$, which is a single point in space. Reflections from all points on the scattering surface come to a focus in the radargram. The amplitude factor in (33) reduces to

$$
\frac{1}{\sqrt{2}} \cdot \frac{S(X) \cdot R}{\left(R^{2}-(X-R)^{2}\right)^{1 / 2}} .
$$

\section{B. Stationary Phase Image of a Point Scatterer}

We now consider a single point scatterer at position $\left(X_{0}, Z_{0}\right)$. The geology is given by

$$
v(X, Z, 0)=S_{0} \delta\left(X-X_{0}\right) \delta\left(Z-Z_{0}\right)
$$

with corresponding 2-D Fourier transform

$$
V(k, \omega, 0)=S_{0} \exp \left(-i\left(k X_{0}-\omega Z_{0}\right)\right) .
$$


Substituting into (21), we obtain for the radar image

$$
u(x, 0, z)=g(z) * \int_{-\infty}^{\infty} S_{0}|\omega| d \omega \int_{-\infty}^{\infty} \exp (i \omega \phi(p)) d p .
$$

The one-dimensional (1-D) stationary phase solution of this integral is

$$
u(x, 0, z)=\frac{1}{\sqrt{2 \pi z}} f(z-H(x))
$$

where

$$
\begin{aligned}
H(x) & =\left[\left(x-X_{0}\right)^{2}+Z_{0}^{2}\right]^{1 / 2} \\
f(z) & =D_{1 / 2}(z) * g(z) .
\end{aligned}
$$

The half-differentiating filter $D_{1 / 2}(z)$, which is convolved with the source pulse $g(z)$, has a Fourier transform $(i \omega)^{1 / 2}$. Therefore, the received scattered radar pulse is out of phase by $\pi / 4$ at all frequencies with respect to the incident transmitted pulse and has its spectral amplitudes boosted as the square root of the frequency. The amplitude also falls off as the inverse square root of distance, in accordance with cylindrical spreading in the 2-D situation.

\section{Stationary Phase Image of a Terminating Reflector}

We next consider a terminating reflector of finite length, which extends from $X=X_{0}$ to $X=X_{1}$. The reflector has the functional form $Z=F(X)$ and reflectivity $S(X)$, which varies along its length. The spectral integral (25) now becomes

$$
\begin{aligned}
u(x, 0, z)=g(z) & * \frac{1}{4 \pi^{2}} \int_{-\infty}^{\infty}|\omega| d \omega \\
& \cdot \int_{-\infty}^{\infty} \int_{X_{0}}^{X_{1}} S(X) \exp (-i \omega \phi(p, X)) d X d p .
\end{aligned}
$$

We have already dealt with the reflection part of the response between the two endpoints. In addition, there are diffractions to consider.

The stationary phase image from the endpoint $X=X_{0}$ is

$$
u(x, 0, z)=+\sqrt{\frac{z}{\pi}} \frac{1}{K} f(z-h(x))
$$

where

$$
\begin{aligned}
h(x) & =\left[\left(x-X_{0}\right)^{2}+Z_{0}^{2}\right]^{1 / 2} \\
K & =x-X_{0}-Z_{0} Z_{0}^{\prime} \\
f(z) & =D_{-1 / 2}(z) * g(z) .
\end{aligned}
$$

Here, $Z_{0}$ and $Z_{0}^{\prime}$ are the depth and gradient of the interface, respectively, at point $X_{0}$. Equation (41) shows that the moveout pattern of the edge diffraction in $x-z$ space is hyperbolic with the apex at the point $\left(X_{0}, Z_{0}\right)$. The transmitted pulse $g(z)$ gets convolved with a negative half-differentiating filter $D_{1 / 2}(z)$
TABLE I

FORM OF THE PULSE FOR GEORADAR MODELING

\begin{tabular}{ll}
\hline \hline \multicolumn{1}{c}{ Feature } & \multicolumn{1}{c}{ Received Signal } \\
\hline reflection & $g(t)$ \\
reverse branch & $h(t)^{*} g(t)$ \\
point scatterer & $D_{1 / 2}(t)^{*} g(t)$ \\
edge diffraction & $\pm D_{-1 / 2}(t)^{*} g(t)$ \\
caustic & $D_{1 / 6}(t)^{*} g(t)$ \\
\hline \hline & where: \\
$D(t)$ is the differentiating filter given by $D_{\alpha}(t) \Leftrightarrow(i \omega)$ a Fourier \\
transform pair, \\
$h(t)$ is the Hilbert transform operator \\
and * denotes convolution. \\
\hline \hline
\end{tabular}

having Fourier transform $(i \omega)^{1 / 2}$. Thus, there is a $-\pi / 4$ phase shift and high-frequency enhancement of the diffracted signal.

There is also an edge diffraction from the other end of the reflector $X=X_{1}$. To obtain its form on the radar image, we simply replace the $X_{0}, Z_{0}$, and $Z_{0}^{\prime}$ terms in (41) and (42) with the values for $X_{1}, Z_{1}$, and $Z_{1}^{\prime}$.

\section{Changes in Pulse Shape}

In the previous sections, we derived the radar images for a continuous reflector, a point scatterer, and a terminating reflector. We saw how the amplitude and moveout patterns varied in each case and were dependent on the reflector shape and curvature and the diffractor position. For a gently curving reflector, the received signal has the same shape as the transmitted pulse $g(z)$. However, it gets filtered or modified in all other cases, as summarized in Table I. For strongly curved reflectors, which result in triplications and ray path crossings, the signal along the reverse branch is the Hilbert transform of the original signal. In other words, there is a phase change of $\pi / 2$. At the caustic points, the original pulse gets convolved with a one-sixth differentiating filter. For a point scatterer, the pulse is convolved with a half-differentiating filter, whereas for an edge diffraction, the convolution is with a negative halfdifferentiating filter. The latter two result in phase changes of $+\pi / 4$ and $-\pi / 4$, respectively.

\section{Spectral Migration}

The migration problem is to start with the radar image $u(x, 0, z)$ and compute (or reconstruct) the object space or geology that produced it. We solve this boundary value problem (BVP) by running the clock backward or by downward continuing the observations to find the wavefield at all points in the medium at arbitrary time (or radar depth). We obtain this wavefield $u(X, Z, z)$ by solving the equation

$$
\frac{\partial^{2} u}{\partial Z^{2}}=\frac{\partial^{2} u}{\partial z^{2}}-\frac{\partial^{2} u}{\partial X^{2}} .
$$

The final solution is obtained by setting $z=0$ to get $u(X, Z, 0)$. 
Once again, (43) can be readily solved by taking Fourier transforms. The 2-D transform of the image is simply

$$
U(k, 0, \omega)=\int_{-\infty}^{\infty} \int_{-\infty}^{\infty} U(x, 0, z) \cdot \exp (-i(k x-\omega z)) d x d z .
$$

The solution of (44) in the transform domain is

$$
U(k, Z, \omega)=U(k, 0, \omega) \cdot \exp \left(-i \sqrt{\omega^{2}-k^{2}} Z\right) .
$$

By taking the 2-D-inverse transform of this expression and setting $z=0$, we obtain the migrated section as

$$
\begin{aligned}
u(X, Z, 0)=\frac{1}{4 \pi^{2}} \int_{-\infty}^{\infty} \int_{-\infty}^{\infty} U(k, 0, \omega) \\
\cdot \exp \left(i\left(k X-\sqrt{\omega^{2}-k^{2}} Z\right)\right) d \omega d k .
\end{aligned}
$$

This solution can be contrasted with Kirchhoff migration [10] in the spatial domain where

$$
\begin{aligned}
u(X, Z, 0)= & \frac{1}{4 \pi^{2}} \int_{-\infty}^{\infty} \int_{-\infty}^{\infty} \int_{-\infty}^{\infty} \int_{-\infty}^{\infty} u(x, 0, z) \\
& \cdot \exp (i[k(X-x) \\
& \left.\left.\quad-\sqrt{\omega^{2}-k^{2}} Z+\omega z\right]\right) d \omega d k d x d z \\
= & \int_{-\infty}^{\infty} \int_{-\infty}^{\infty} u(x, 0, z) \frac{\partial}{\partial Z}\left[\frac{-1}{\pi} \frac{H(z-r)}{\sqrt{z^{2}-r^{2}}}\right] d x d z
\end{aligned}
$$

where

$$
r^{2}=Z^{2}+(X-x)^{2}
$$

and $H$ is the Heaviside function. The curve $z=r$ is the diffraction hyperbola.

\section{A. Stationary Phase Migration of Radar Section $z=h(x)$}

The integral of (47) can be approximated using the method of stationary phase, just as we did for the solving the forward problem. Consider an event in the image space that has the functional form $z=h(x)$. Let the amplitude vary with the horizontal coordinate according to $T(x)$, and let the wavelet be of the form $g(z)$. We can then represent this event by the expression

$$
u(x, 0, z)=T(x) \delta(z-h(x)) * g(z) .
$$

The stationary phase solution is

$$
u(X, Z, 0)=|\xi|^{-1 / 2} T(x) \hat{g}[\phi(p, x)]
$$

where

$$
\xi=\left[1-\frac{Z}{\sigma}\right] \quad \sigma=\left[\frac{\left(1-h^{\prime 2}\right)^{3 / 2}}{h^{\prime \prime}(x)}\right] .
$$

The point $(p, x)$ is a stationary point if

$$
\phi(p, x)=-p(X-x)+\sqrt{1-p^{2}} Z-h(x) .
$$

Near $\phi=0$, we have

$$
\begin{aligned}
& X=x-h(x) h^{\prime}(x) \\
& Z=h(x) \sqrt{1+h^{\prime}(x)^{2}} .
\end{aligned}
$$

These parametric equations for the object space correspond to the Cartesian equation $Z=F(X)$.

Thus, finally, we obtain the stationary phase solution to the migration problem as

$$
u(X, Z, 0) \approx T(x)|\xi|^{-1 / 2} \phi_{Z} \hat{g}[(Z-F(X)]
$$

where

$$
\phi_{Z}=\frac{d \phi}{d Z}=\sqrt{1-h^{\prime}(x)^{2}}<1
$$

and the hat on $g$ has the same meaning as in (27), viz., take the Hilbert transform if $\xi$ is negative.

\section{B. Numerical Example}

We now apply the theory to a simple numerical example. The radar section (image) shown in Fig. 7 comprises 80 traces at 1 -m spacing. It shows a curved reflector having both an anticlinal segment and a synclinal segment. The pulse $g(t)$ is a single cycle sinusoid of frequency $150 \mathrm{MHz}$ (wavelength $1 \mathrm{~m}$ in a medium of dielectric constant 4), having constant amplitude across the section, i.e., $T(x)=1$. For each value of $x$, we can estimate $h(x)$ and the local gradient $h^{\prime}(x)$ numerically. Thus, we can calculate the coordinates in object space $(X, Z)$ of each point $(x, z)$. The "reflectivity" at this point is given by the composite amplitude factor in (55). This depends on the numerical values for $T(x), \xi(x)$, and $\phi_{z}(x)$. The latter is solely a function of $h^{\prime}(x)$, whereas $\xi(x)$ depends on the function $h(x)$ as well as its first and second derivatives. The derivatives $h^{\prime}(x)$ and $h^{\prime \prime}(x)$ can be readily obtained through the use of spline functions or some other numerical differentiating procedure. The resulting migrated section (geological model) is shown as the dotted line in Fig. 7, superimposed on the radar image. Each dot can be associated with a particular trace (wavelet) in the image.

The numbers at spot locations indicate the deduced reflectivity values of the interface, which vary smoothly across the section. Note how reflected pulses from lower reflectivity portions of the interface (e.g., at the syncline) have equal amplitude to those pulses reflected from higher reflectivity portions of the interface (at the top of the anticline). This is 


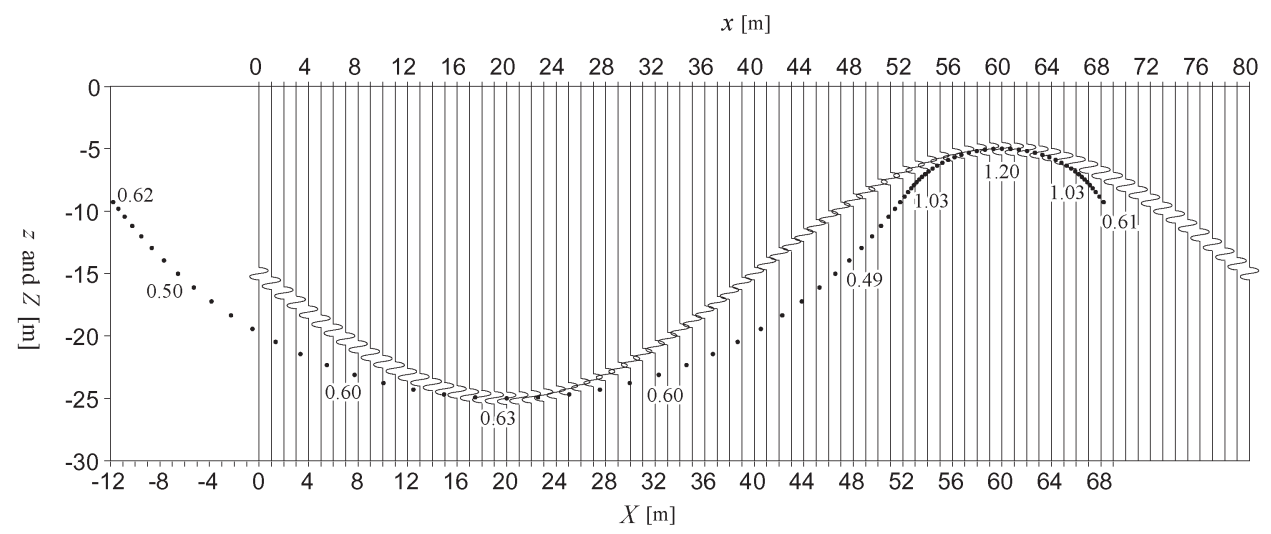

Fig. 7. Numerical example with a curved reflector having both an anticlinal segment and a synclinal segment. The radar section is composed of 80 traces ( $x$ axis). The resulting migrated section (geological model) is shown as the dotted line ( $X$ axis). The deduced reflectivity values are shown at spot locations along the profile.

a clear demonstration of how rays get focused or defocused by bed curvature. Convergence (increased amplitude) occurs at synclines, whereas divergence (reduced amplitude) occurs at anticlines. In this synthetic example, all wavelets in the image were assigned equal amplitude; thus, the reflectivity had to vary to accommodate this. For clarity of presentation, we have drawn the interface as a sharp line (series of dots) rather than a series of migrated wavelets. In reality, the resolution is limited by the bandwidth of the pulse $g(z)$ and level of noise. For this particular situation, the pulse shape does not change because the amplitude factor $\xi$ remains positive. Note how the anticline is narrowed in its width and the syncline is broadened on the migrated section, although the peak and trough remain in the same spatial position.

The accuracy of this procedure depends on a number of factors, principally the level of data noise and how well the pulse onset time, amplitude, and shape can be extracted from each trace of the radar section. Furthermore, it should be appreciated that the multidimensional stationary phase technique for evaluating the integrals is an approximate procedure. It is strictly the high-frequency solution; thus, it is implicit in the analysis that the GPR wavelength is small relative to the reflector depth and lateral dimension of the structure being imaged.

\section{CONCLUSION}

Using the method of stationary phase, we have evaluated the infinite spectral integrals to obtain explicit expressions for the zero-offset georadar response of an arbitrary shaped 2-D reflector of variable reflectivity, a point scatterer, and a terminating reflector, as well the migration of arbitrary events on the GPR section. The resulting equations combine the kinematic properties of the image (geometry of transmitters and receivers and structure) with the dynamic (amplitude and phase) information. The theoretical formulation, although restricted to constant average wavespeed models, shows the influence of reflector gradient and curvature on the radar signal. For multiple reflectors and a model in which velocity varies with depth, one would have to use a different average wavespeed above each reflector. The migration equation accommodates amplitude and phase fluctuations along the radar section, including the effects of cusps and caustics. It is suitable as a first approximation (section migration) to imaging noisy data to obtain the shape and form of a reflector where standard wavefield migration techniques may fail.

\section{ACKNOWLEDGMENT}

The authors would like to thank A. Green for reviewing this paper.

\section{REFERENCES}

[1] M. Gransmueck and A. G. Green, "3-D georadar mapping: Looking into the subsurface," Environ. Eng. Geosci., vol. 2, no. 2, pp. 195-200, May 1996.

[2] J. Tronicke, P. Villamar, and A. G. Green, "Detailed shallow geometry and displacement estimates of the Maleme Fault Zone, New Zealand, using 2-D and 3-D georadar," Near Surf. Geophys., vol. 4, June 2006.

[3] M. Beres, P. Huggenberger, A. G. Green, and H. Hortsmeyer, "A study of glaciofluvial architecture using two and three-dimensional georadar methods," Sediment. Geol., vol. 129, no. 1/2, pp. 1-24, Nov. 1999.

[4] M. Gransmueck, R. Weger, and H. Horstmeyer, "Three-dimensional ground penetrating radar imaging of sedimentary structure, fracture and archaeological features of submeter resolution," Geology, vol. 32, no. 11, pp. 933-936, Nov. 2004.

[5] R. Birke and R. Versteeg, "Use of four-dimensional ground penetrating radar and advanced visualization methods to determine subsurface fluid migration," J. Appl. Geophys., vol. 43, no. 2-4, pp. 215-226, Mar. 2000.

[6] B. Whiting, D. McFarlan, and S. Hackenberger, "Three-dimensional GPR study of a prehistoric site in Barbados, West Indies," J. Appl. Geophys., vol. 47, no. 3/4, pp. 217-226, Jul. 2001.

[7] M. Gransmueck, R. Weger, and H. Horstmeyer, "Full-resolution 3-D GPR imaging," Geophysics, vol. 70, no. 1, pp. K-12-K-19, Jan./Feb. 2005.

[8] S. Wänstedt, S. Carlsten, and S. Tiren, "Borehole radar measurements aid structure geological interpretations," J. Appl. Geophys., vol. 43, no. 2-4, pp. 227-237, Mar. 2000.

[9] A. Binley, P. Winship, R. Middleton, M. Pokar, and J. West, "Highresolution characterization of vadose zone dynamics using cross-borehole radar," Water Resour. Res., vol. 37, no. 11, pp. 2639-2652, Nov. 2001.

[10] A. J. Berkhout, Seismic Migration: Imaging of Acoustic Energy by Wavefield Extrapolation. Part A. Theoretical Aspect. Amsterdam, The Netherlands: Elsevier, 1982.

[11] J. F. Claerbout, Imaging the Earth's Interior. Oxford, U.K.: Blackwell, 1985.

[12] E. Fisher, G. A. McMechan, P. Annan, and S. W. Cosway, "Examples of reverse-time migration of single-channel ground penetrating radar profiles," Geophysics, vol. 57, no. 4, pp. 577-586, Apr. 1992.

[13] F. Lehmann and A. G. Green, "Topographic migration of georadar data: Implications for acquisition and processing," Geophysics, vol. 65, no. 3, pp. 836-848, May/Jun. 2000. 
[14] H. Zhou, M. Sato, and H. Liu, "Migration velocity analysis and prestack migration of common-transmitter GPR data," IEEE Trans. Geosci. Remote Sens., vol. 43, no. 1, pp. 86-91, Jan. 2005.

[15] J. van der Kruk, C. P. A. Wapenaar, J. T. Fokkema, and P. M. van der Berg, "Three-dimensional imaging of multicomponent ground-penetrating radar data," Geophysics, vol. 68, no. 4, pp. 1241-1254, Jul./Aug. 2003.

[16] J. Zhe and S. A. Greenhalgh, "A new kinematic method for mapping seismic reflectors," Geophysics, vol. 64, no. 5, pp. 1594-1602, Sep./Oct. 1999.

[17] N. G. Van Kampen, "An asymptotic treatment of diffraction problems," Physica, vol. 14, no. 9, pp. 575-589, Jan. 1949.

[18] _ "The method of stationary phase and the method of Fresnel zones," Physica, vol. 24, no. 6, pp. 437-444, 1958

[19] D. S. Jones and M. Kline, "Asymptotic expansion of multiple integrals and the method of stationary phase," J. Math. Phys., vol. 37, pp. 1-28, 1958.

[20] S. A. Greenhalgh, D. R. Pant, and C. R. A. Rao, "Effect of reflector shape on seismic amplitude and phase," Wave Motion, vol. 16, no. 4, pp. 307322, Dec. 1992.

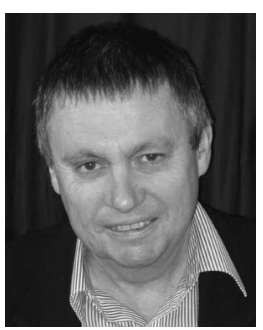

Stewart A. Greenhalgh received the B.Sc., M.Sc., and D.Sc. degrees from Sydney University, Sydney, Australia, in 1972, 1976, and 1997 respectively, and the Ph.D. degree from the University of Minnesota, Minneapolis, in 1979.

He is currently a Professor of geophysics with the Department of Physics, Adelaide University, Adelaide, Australia. He has worked as a professional or consultant in various areas of mining, petroleum, and engineering geophysics, as well as having been an academic for over 20 years. He has published widely in both experimental and theoretical geophysics. His primary research interests are in seismic and electrical exploration.

Dr. Greenhalgh is a Fellow of the Australian Academy of Technological Sciences and Engineering, a Fellow of the Institute of Physics, U.K., and a member of the American Geophysical Union, the Society of Exploration Geophysicists, the European Association of Geoscientists and Engineers, and the Seismological Society of America.

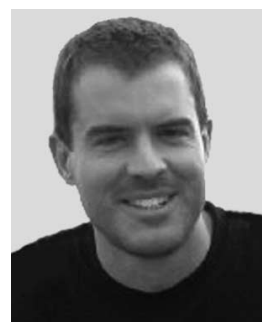

Laurent Marescot received diplomas in geophysics and geology from the University of Lausanne, Lausanne, Switzerland, in 1998 and 2000, respectively, and the $\mathrm{Ph} . \mathrm{D}$. degree in geophysics from the University of Nantes, Nantes, France and the University of Lausanne, in 2004.

$\mathrm{He}$ is a Researcher with the Applied and Environmental Geophysics Group, Swiss Federal Institute of Technology (ETHZ), Zurich, Switzerland. His primary research interests are in electromagnetic methods, finite-element modeling, and inversion strategies. He is mainly involved in electrical resistivity imaging for hydrogeophysics, civil engineering studies, and nondestructive testing.

Dr. Marescot is a member of the Society of Exploration Geophysicists, the European Association of Geoscientists and Engineers, and the Environmental and Engineering Geophysical Society. 